\title{
Seguimiento de lactantes hospitalizados por bronquiolitis por virus respiratorio sincicial. Evolución clínica, respuesta de atopia inflamatoria y marcadores. Resultados preliminares
}

\author{
GUILLERMO ZEPEDA F.*, PATRICIA DÍAZ A.**, RICARDO PINTO M.*, \\ ALDO GAGGERO B.*** y PAOLA UASAPUD E.****
}

Follow-up of infants hospitalized for bronchiolitis by respiratory syncytial virus. Clinical evolution, inflammatory response and markers of atopy. Preliminary results

Background: Respiratory syncytial virus infection (RSV) alone or associated to rhinovirus (RV) in the infant has been linked with more likelihood to develop asthma and atopy. Aim: Analyze clinical and immunological markers of patients with RSV or RV bronchiolitis that determine their evolution. Patients and Methods: We studied previously healthy infants hospitalized for bronchiolitis during the fall-winter period of 2009 and 2010. RSV and RV by qPCR, and proinflammatory interleukins (IL). IL-6, $I L-8, T N F-\alpha, I L-1 \beta$ and IL-12, were determined in nasopharyngeal aspirate (NPA). A follow-up clinical, indoor pollution and immunological study was done at 4 or 5 years. Results are expressed in median and range. Mann-Whitney's test was used in the nonparametric statistical analysis. Results: Eight out of 22 patients (36\%) are currently with recurrent wheezing $(R W)$ in treatment with budesonide $400 \mu \mathrm{g}$ per day as a mean dose. In the IL assessment significant changes were detected only in IL-1 $\beta$ that was increased and in $I L-12$ that was decreased in the $R W$ group versus the non $R W(N R W)$ group. There were not significant differences in both groups in age at hospitalization, infection severity, presence of personal or family atopy, co-infection with RSV and RV, presence of older siblings or indoor air pollution. Conclusions: The determination of IL-1 $\beta$ and IL-12 in NPA for bronchiolitis could be an early marker of subsequent inflammation of the airway. Co-infection of RSV and RV does not get worse the clinical evolution. The group $R W$ of preschool children had no further development of atopy than the $N R W$ group. There could be other factors that contribute to the manifestation of bronchial inflammation in the $R W$ group.

Key words: bronchiolitis, respiratory syncytial virus, rhinovirus, interleukins, follow up, recurrent wheezing, asthma.

\section{Resumen}

Introducción: Se ha relacionado la infección por Virus Respiratorio Sincicial (VRS) solo o asociado a Rinovirus $(R V)$ en el período de lactante con mayor probabilidad de desarrollar atopia y asma. Objetivo: Analizar marcadores clínicos e inmunológicos de pacientes con bronquiolitis por VRS y/o RV que determinen su evolución. Material y Método: Lactantes previamente sanos hospitalizados por bronquiolitis, en el hospital Roberto del Río en el periodo de otoño-invierno de 2009 y 2010. Se determinó en aspirado nasofaríngeo (ANF) VRS y RV por qPCR, e interleuquinas (IL) proinflamatorias (IL-6, $I L-8, T N F-\alpha, I L-1 \beta$ e IL-12). Seguimiento clínico y estudio inmunológico a los 4 o 5 años. Los resultados se expresan en medianas y rango. Análisis estadístico no paramétrico con test de Mann-Whitney.

\footnotetext{
* Departamento de Pediatría y Cirugía Infantil, Facultad de Medicina, Campus Norte, Universidad de Chile. Hospital Roberto del Río.

** Programa de Fisiopatología, Instituto de Ciencias Biomédicas, Facultad de Medicina, Universidad de Chile.

*** Programa de Virología, Instituto de Ciencias Biomédicas, Facultad de Medicina, Universidad de Chile.

**** Médico-Cirujano. Centro de Salud Familiar Agustín Cruz Melo.
} 
Resultados: 22 pacientes seguidos hasta ahora, 8 (36\%) son actualmente sibilantes recurrentes (SR) en tratamiento con budesonida dosis mediana de $400 \mu \mathrm{g} /$ día. De las ILs evaluadas sólo la elevación de la $I L-1 \beta$ y la disminución de la IL-12 se objetivaron con diferencias significativas en el grupo de SR versus el grupo No SR. No hubo diferencias significativas en estos dos grupos en edad de hospitalización, gravedad de la infección, presencia de atopia personal o familiar, coinfección de VRS y RV, presencia de hermanos mayores ni contaminación intradomiciliaria. Conclusiones: La determinación de IL-1 $\beta$ y de IL-12 en ANF durante la bronquiolitis podría ser un marcador precoz de inflamación posterior de la vía aérea. La co-infección de VRS y RV no empeora la evolución clínica. Este grupo de preescolares SR no tiene mayor desarrollo de atopia que los no SR. En este grupo de SR podrían existir otros factores que ayuden a contribuir a la manifestación de inflamación bronquial.

Palabras clave: Bronquiolitis, virus respiratorio sincicial, rinovirus, interleukinas, seguimiento, sibilancias recurrentes, asma.

\section{Introducción}

Según el Departamento de Estadísticas e Información de Salud (DEIS) del Ministerio de Salud de Chile en su último registro que data del año 2011 la principal causa de hospitalización en los menores de 10 años es la infección respiratoria aguda baja representando el $31,4 \%$ del total de hospitalizaciones ${ }^{1}$, siendo las causas virales y especialmente el virus respiratorio sincicial (VRS) el agente más frecuentemente involucrado ${ }^{2}$. Esta realidad es similar a otros países, en los cuales el uso de técnicas moleculares permite identificar la mayoría de los agentes patógenos virales involucrados en bronquiolitis siendo los principales el VRS y el rinovirus $(\mathrm{RV})^{3,4}$.

Estos dos virus causantes de bronquiolitis en el período de lactante, se han asociado con atopia y/o asma en etapas posteriores en cohortes de alto riesgo de asma o determinados por su gravedad clínica $^{3,5,6}$. Aún no están claros los mecanismos etiopatogénicos que traducen estas asociaciones.

Considerando estos antecedentes, el objetivo de nuestro estudio es evaluar marcadores clínicos e inmunológicos de pacientes con bronquiolitis por VRS y/o RV que determinen su evolución y de esta manera poder identificar pacientes que pudieran tener riesgo de sufrir de asma y/o sibilancias recurrentes en etapas posteriores.

\section{Metodología}

\section{Pacientes}

Se reclutaron pacientes menores de un año internados en el Hospital Roberto del Río por bronquiolitis por VRS detectados por inmunofluorescencia indirecta (IFI) durante los meses de otoño e invierno de los años 2009 y 2010. Cabe destacar, que este estudio continúa con el seguimiento y que se ingresaron nuevos pacientes hasta el año 2013, es decir, 5 años seguidos (des- de 2009 a 2013) y que para fines de este trabajo sólo se incluyeron los pacientes que ya hubieran cumplido 4 o 5 años de vida. Se excluyeron los menores de 1 mes, comorbilidad importante, antecedente de prematurez y uso de corticoides previos, en cualquiera de sus vías de administración. El estudio fue aprobado por los comités de ética del Servicio de Salud Metropolitano Norte y de la Facultad de Medicina de la Universidad de Chile. Los padres firmaron el consentimiento informado para que sus hijos pudieran ingresar al estudio.

Para realizar el seguimiento, se contactó a los pacientes por vía telefónica $\mathrm{y} / \mathrm{o}$ visita domiciliaria durante el primer semestre del año 2014 y se les citó para que asistieran al Hospital Roberto del Río para proseguir con el estudio.

Las muestras de sangre y de aspirado nasofaríngeo (ANF) de los pacientes se obtuvieron al ingreso del estudio (hospitalización) y en el seguimiento realizado a los 4 o 5 años.

\section{Datos de hospitalización}

Obtuvimos de la ficha clínica y de la evaluación hospitalaria los siguientes datos: edad, sexo, días de hospitalización, días de oxígeno, requerimiento máximo de oxígeno, tipo de virus detectado, signos de atopia detectados al ingreso, número de hermanos, antecedente familiar de asma, dermatitis atópica o rinitis alérgica, asistencia a sala cuna, tabaquismo familiar y tipo de calefacción. El registro se realizó mediante una planilla Excel.

\section{Aspirado nasofaríngeo}

Con una sonda Intube de 8 Fr de diámetro se aspiró la secreción nasofaríngea y se colocó en 3 $\mathrm{ml}$ de solución salina en un recipiente refrigerado para su traslado al laboratotrio de virología. Una vez agitada y homogeneizada la muestra se fraccionó para realizar el estudio virológico. El sobrenadante se guardó a $-80^{\circ} \mathrm{C}$ para cuantificar citoquinas. 


\section{Estudio virológico}

Se estudió la presencia de VRS y RV por PCR (reacción en cadena de la polimerasa) en tiempo real (qPCR).

\section{Cuantificación de citoquinas}

La concentración de citoquinas en el ANF fue determinada usando un kit de CBA "Cytokine Beads Assay" (BD Biosciences) de acuerdo al protocolo indicado por el fabricante. Evaluamos las concentraciones de: IL-6, IL-8, TNF- $\alpha$ (factor de necrosis tumoral alfa), IL-1 $\beta$ e IL-12 mediante citómetro de flujo (FAST Can to II con software DIVA).

\section{Entrevista de seguimiento}

Se elaboró una pauta de entrevista para evaluar la evolución de los pacientes consignando los siguientes datos: diagnóstico de asma o SBO recurrente realizado por un médico, controles periódicos en consultorio y/o hospital por cuadros bronquiales repetidos, uso de corticoides inhalatorios permanentes, diagnóstico médico de rinitis alérgica y/o dermatitis atópica, respiración bucal o roncopatía, uso de prednisona indicado por un médico, silbidos al pecho, tabaquismo familiar, tipo de calefacción, número de hermanos y asistencia a jardín infantil y/o colegio.

\section{Muestra de sangre}

Se obtuvo $5 \mathrm{ml}$ de sangre la que se heparinizó y se le extrajo el plasma para cuantificar IgE total.

\section{Prick test}

A los niños que se les realizó el seguimiento se les practicó un prick test en la cara anterior del antebrazo solicitándose la suspensión de la administración de antihistamínicos 7 días antes en caso de estar recibiendo este tipo de tratamiento. Este procedimiento lo realizó una tecnóloga- médico o técnico en enfermería ambas entrenadas en esta técnica. Se utilizaron los siguientes alergenos inhalatorios: Dermatophagoides farinae, Dermatophagoides pteronyssinus, mezcla de epitelio de animales (perro, gato, caballo), mezcla de hongos (Alternaria alternata, Penicillium notatum, Aspergillus oryzae, Cladosporium sphaerospermum, Chrysogenum), mezcla de pólenes de malezas (ligustrina, acedera, diente de león), de pastos (ballica, poa, festuca), de árboles (plátano oriental, álamo, abedul, arce) y Aspergillus fumigatus. Siguiendo las recomendaciones internacionales el control positivo se realizó con histamina $10 \mathrm{mg} /$ $\mathrm{ml}$ y el control negativo con solución salina, la lectura del examen se realizó a los 15 min de realizado éste y fueron consideradas como resultado positivo las pápulas mayores o iguales a $3 \mathrm{~mm}$ de diámetro ${ }^{7}$.

\section{IgE total}

De la muestra de sangre se extrajo plasma para la determinación de IgE total mediante la prueba VIDAS ${ }^{\circ}$ usando la técnica ELFA (Enzyme Linked Fluorescent Assay).

\section{Análisis estadístico}

Los resultados se expresan como números absolutos, promedios o medianas y rangos según se explícita en el texto y se analizaron aplicando el test no paramétrico de Mann-Whitney. Un valor de $\mathrm{p}<0,05$ se consideró significativo.

\section{Resultados}

Del total de 22 pacientes seguidos hasta ahora (niños que han cumplido 4 o 5 años de vida), 15 son hombres y actualmente $8(36 \%)$ son sibilantes recurrentes (SR) y 14 son no sibilantes recurrentes (NSR). De los SR, 6 de ellos se encuentran recibiendo tratamiento con budesonida inhalatoria $400 \mu \mathrm{g} /$ día como promedio con un rango de 200 a $600 \mu \mathrm{g} /$ día.

A continuación se detallarán los resultados según hospitalización o seguimiento:

\section{Resultados durante hospitalización (Tabla 1)}

La edad de hospitalización y por ende, de ingreso al estudio, fue de 4,0 meses en promedio con un rango de 1 a 9 meses para la serie SR versus 4,5 meses con un rango de 1 a 10 meses para la serie NSR.

La gravedad clínica determinada por los días de hospitalización, días de oxígenoterapia y máximo flujo de oxígeno $(\mathrm{L} / \mathrm{min})$ fue similar en ambos grupos.

Los signos clínicos de atopia personal (dermatitis atópica y/o alergia alimentaria y/o rinitis alérgica) no se registraron en ningún paciente del grupo SR versus un paciente en el grupo NSR que presentaba criterios clínicos de dermatitis atópica.

La historia familiar de atopia (asma y/o rinitis alérgica y/o dermatitis atópica) se registró en 5 pacientes del grupo SR versus 4 pacientes del grupo NSR.

La presencia de hermanos mayores se registró en 4 pacientes del grupo de SR versus 7 pacientes del grupo de NSR.

La contaminación intradomiciliaria en relación a tabaquismo familiar y calefacción por combustión (leña y/o brasero y/o estufa a parafina y/o estufa a gas) estuvo presente en el grupo de 
SR en 6 y 6 pacientes respectivamente, mientras que en el grupo NSR estuvo presente en 8 y 10 pacientes. Se destaca el alto nivel de tabaquismo en ambos grupos, del total de pacientes 64\% $(14 / 22)$ fumaba por lo menos un integrante de la familia, siendo el más frecuente el padre con un $71 \%(10 / 14)$.

Del estudio viral destaca la presencia de VRS como único agente en 13 casos, 5 casos de SR versus 8 de NSR. La co-infección por VRS y rinovirus destaca en 9 pacientes, siendo en 3 de los SR versus 6 de los NSR.
De todas las citoquinas estudiadas (IL-6, IL-8, TNF- $\alpha$, IL-1 $\beta$ e IL-12) sólo las IL-1 $\beta$ y la IL 12 , con elevación y disminución respectivamente, mostraron una diferencia estadísticamente significativamente en el grupo SR versus el grupo NSR.

\section{Resultados durante el seguimiento (Tabla 2)}

La edad promedio de los pacientes durante el seguimiento correspondió a 4,7 años para la serie SR versus 4,8 años para la serie NSR.

La presencia de hermanos en el grupo de SR se

Tabla 1. Características clínicas e inmunológicas durante su hospitalización de los pacientes que evolucionaron con y sin sibilancias recurrentes

\begin{tabular}{|c|c|c|c|}
\hline & $\begin{array}{l}\text { Sibilantes recurrentes } \\
\qquad(\mathbf{n}=\mathbf{8})\end{array}$ & $\begin{array}{l}\text { No sibilantes recurrentes } \\
\qquad(\mathrm{n}=14)\end{array}$ & $\mathbf{p}$ \\
\hline Género & $30^{\lambda} \quad 5$ ㅇ & $12 \hat{0} \quad 2$ ㅇ & \\
\hline Edad hospitalización (meses)* & $4,0 \quad(1-9)$ & $4,5(1-10)$ & NS \\
\hline Hospitalización (días)* & $6,4(2-12)$ & $5,9(3-12)$ & NS \\
\hline Oxígenoterapia (días)* & $4,6 \quad(0-8)$ & $3,7(0-10)$ & NS \\
\hline Flujo máximo $\mathrm{O}_{2}(\mathrm{~L} / \mathrm{min})^{*}$ & $1 \quad(0-2)$ & $1 \quad(0-2)$ & NS \\
\hline Signos de atopia personal ${ }^{* *}$ & 0 & 1 & NS \\
\hline Atopia familiar** & 5 & 4 & NS \\
\hline Hermanos** & 4 & 7 & NS \\
\hline Tabaquismo** & 6 & 8 & NS \\
\hline Calefacción por combustión** & 6 & 10 & NS \\
\hline VRS (único)** & 5 & 8 & NS \\
\hline $\mathrm{VRS}+\mathrm{RV}^{* *}$ & 3 & 6 & NS \\
\hline IL $-1 \beta \mathrm{pg} / \mathrm{ml}(\mathrm{ANF})^{* * *}$ & $1.447(107-8.500)$ & $107(1,4-3.217)$ & $<0,04$ \\
\hline $\mathrm{IL}-12 \mathrm{pg} / \mathrm{ml}(\mathrm{ANF})^{* * *}$ & $0,0(0-3,5)$ & $3,5(0-20,7)$ & $<0,04$ \\
\hline
\end{tabular}

*Datos expresados como promedios y rangos mínimo y máximo. **Datos expresados como números absolutos. ***Datos expresados como medianas y rangos mínimo y máximo. ANF: aspirado naso-faríngeo.

Tabla 2. Características demográficas e inmunológicas y contaminación intradomiciliaria al seguimiento a 4 o 5 años de los 22 pacientes estudiados

\begin{tabular}{|c|c|c|c|}
\hline & $\begin{array}{l}\text { Sibilantes recurrentes } \\
\qquad(\mathrm{n}=\mathbf{8})\end{array}$ & $\begin{array}{l}\text { No Sibilantes recurrentes } \\
\qquad(\mathrm{n}=14)\end{array}$ & $\mathbf{p}$ \\
\hline Edad (años)* & 4,7 & 4,8 & \\
\hline Hermanos $(\mathrm{n})^{* *}$ & $5(63 \%)$ & $8(57 \%)$ & NS \\
\hline Tabaquismo familiar** & $6(75 \%)$ & $10(71 \%)$ & NS \\
\hline Calefacción por combustión & $5(63 \%)$ & $7(50 \%)$ & NS \\
\hline Atopia familiar** & $5(63 \%)$ & $4(29 \%)$ & NS \\
\hline Atopia paciente** & $5(63 \%)$ & $3(21 \%)$ & NS \\
\hline Prick test positivos** & $3(38 \%)$ & $6(43 \%)$ & NS \\
\hline IgE total $\mathrm{UI} / \mathrm{ml} * * *$ & $28(4-521)$ & $36(1-769)$ & NS \\
\hline
\end{tabular}

*Datos expresados como promedio. **Datos expresados como número absoluto. ***Datos expresados como mediana y rangos mínimo y máximo. 
objetivó en 5 pacientes (63\%) y en el grupo NSR en 8 pacientes $(57 \%)$.

La contaminación intradomiciliaria evaluada como tabaquismo familiar y uso de calefacción por combustión no detectó diferencias significativas en ambos grupos.

La atopia familiar se mantuvo en los mismos valores que durante la hospitalización, ya que, con la llegada de nuevos hermanos no hubo aumento de estos valores.

La atopia personal objetivada con prick test positivo para inhalantes no se encontró diferencia entre el grupo de SR versus el grupo NSR.

Los niveles de IgE total no evidenciaron diferencias significativas entre ambos grupos.

\section{Discusión}

Se ha relacionado tanto a la bronquiolitis causada por VRS como a la causada por RV con la probabilidad de desarrollar asma y/o sibilancias recurrentes en etapas posteriores en diferentes cohortes, tanto con pacientes seguidos desde el nacimiento con niños de alto riesgo de asma debido a la presencia de atopia en al menos uno de los padres ${ }^{6,8}$ como en cohortes determinadas por la gravedad de la infección inicial debido al criterio de selección de bronquiolitis hospitalizada $^{4,5}$. En nuestros pacientes seguidos por 4 o 5 años no apreciamos un aumento significativo del riesgo de ser asmático y/o sibilante recurrente en relación a la presencia de co-infección VRS-RV. Este punto es discordante a lo relatado previamente en que se describe un odds ratio (razón de disparidad) $\mathrm{OD}=10$ de ser asmático a los 6 años en una cohorte de alto riesgo al presentar una coinfección VRS-RV antes de los 3 años ${ }^{3}$. Probablemente el hecho que nuestros pacientes no tengan historia de atopia personal ni familiar explicaría en parte esta diferencia, ya que, se ha relacionado a la presencia de atopia previa a la infección por $\mathrm{RV}$ como un factor de riesgo para desarrollar asma en etapas posteriores ${ }^{8,9}$. Este punto cobra mayor importancia al reconocer que el asma atópico es el tipo de asma más frecuente tal como se relata en datos nacionales ${ }^{10}$ como internacionales ${ }^{11}$ y que se ha comunicado la importancia de buscar marcadores tempranos de atopia para identificar los niños sibilantes que tendrán más riesgo de presentar asma persistente ${ }^{12}$. El VRS por su parte se ha postulado que tendría dentro de su etiopatogenia una mayor probabilidad de desarrollar sibilancias recurrentes y/o asma por mecanismos diferentes de la atopia ${ }^{13}$.

Un punto importante es que debido al cri- terio de inclusión principal de nuestro trabajo (bronquiolitis por VRS determinada por IFI) no tuvimos RV como agente etiológico único, pero llama la atención el porcentaje de co-infección con ambos agentes ( $41 \%$ del total), no quedando claro el rol en el cuadro agudo del rinovirus, por lo que este hecho se debe tomar con cautela, ya que, se ha demostrado la presencia de rinovirus inclusive en población asintomática ${ }^{6}$. Más aún, en este trabajo aún no se había serotipificado el tipo de rinovirus, ya que, se ha implicado el rinovirus $\mathrm{C}$ como probablemente más patogénico en comparación a las otras especies ${ }^{14}$.

Previamente habíamos demostrado que las ILs proinflamatorias en ANF y el cortisol plasmático se correlacionaban directamente con la gravedad de la bronquiolitis por $\operatorname{VRS}^{15,16}$ y que durante esta enfermedad se evidenciaba un aumento del receptor $\beta$ de glucocorticoides el cual es inactivo a la acción de los corticoides ${ }^{17}$. Ahora bien, durante nuestro seguimiento evidenciamos que sólo la elevación de IL-1 $\beta$ y la disminución de IL-12 tomadas durante el episodio de bronquiolitis fueron consistentes con una mayor probabilidad de asociación con sibilancias recurrentes a la edad de 4 o 5 años con independencia de la gravedad de la infección, de la contaminación intradomiciliaria, presencia de atopia personal o familiar, entre otros factores, por lo que podrían ser considerados como marcadores precoces de inflamación posterior de la vía aérea. De esta manera, aun cuando el número de pacientes es pequeño y los cambios que puedan experimentar estos marcadores en el tiempo pueden modificar estos resultados, es importante considerarlos como eventuales marcadores biológicos, ya que, están determinados genéticamente e indicarían una forma de respuesta inflamatoria. Así, dentro de los índices predictivos que evalúan diferentes marcadores para asma en lactantes y/o preescolares se ha sabido que tienen mejor valor predictivo negativo que positivo ${ }^{18}$, por lo cual agregar otros elementos como por ejemplo, citoquinas proinflamatorias al ingreso a un paciente con bronquiolitis hospitalizado, nos podría orientar acerca de qué pacientes tuvieran más probabilidad de seguir con sibilancias recurrentes en el período preescolar. En relación a este punto se destaca la realización rutinaria en algunos centros europeos de la determinación de interleukinas para la toma de decisiones clínicas en un marco clínico habitual y no sólo a nivel de investigación.

El alto nivel de contaminación intradomiciliaria al cual son sometidos nuestros pacientes, manifestado tanto como calefacción con métodos de combustión interna como de tabaquismo 
familiar, es sumamente importante en el sentido de que se ha visto una mayor probabilidad de presentar asma en la vida adulta en los adultos con tabaquismo activo que habían presentado una infección respiratoria baja por VRS en la infancia ${ }^{19}$.

Dentro de las limitaciones de este estudio hay que señalar que se trata de una cohorte que discrimina por la gravedad del paciente ya que, al ingreso todos estaban hospitalizados por bronquiolitis. Otras limitaciones son el número pequeño de pacientes actualmente evaluados y no haber serotipificado los pacientes con rinovirus.

Dentro de sus ventajas podemos destacar que es un estudio con seguimiento prolongado de los pacientes de nuestro país y que actualmente sigue en desarrollo, por lo cual, el número de pacientes finalmente estudiados será mayor al presentado en esta comunicación. Otras ventajas son: a) que no se discriminó previo a la hospitalización por lo que no tenemos el sesgo de que nuestros resultados sean sólo aplicables a población con alto riesgo de ser asmáticos; y b) utilizar tanto biología molecular para evaluación de agentes virales como tecnología avanzada para determinar citoquinas.

En resumen, se presentan los resultados preliminares de un estudio que está en marcha y que evalúa un seguimiento de 4 a 5 años de pacientes hospitalizados por bronquiolitis que determina que la elevación de IL-1 $\beta$ y la disminución de IL-12 tomadas del ANF durante el episodio agudo fueron los únicos de los biomarcadores evaluados en que se observó una asociación con la presencia de sibilancias recurrentes en el período preescolar.

\section{Agradecimientos}

Estudio financiado por proyecto Fondecyt 1120411.

\section{Bibliografía}

1.- MINISTERIO DE SALUD. Departamento de Estadística e Información de Salud. Egresos hospitalarios 2011. Disponible en: http://intradeis.minsal.cl/egresoshospitalarios/menu_publica_nueva/menu_publica_nueva.htm (Consultado en marzo de 2015).

2.- MINISTERIO DE SALUD. Guía Clínica Infección Respiratoria Aguda Baja de Manejo Ambulatorio en menores de 5 años. Santiago: Minsal, 2013.

3.- JACKSON D J, GANGNON R E, EVANS M D, ROBERG K A, ANDERSON E L, PAPPAS T E, et al. Wheezing rhinovirus illnesses in early life predict asthma development in high-risk children. Am J Respir Crit Care Med 2008; 178: 667-72.

4.- TAKEYAMA A, HASHIMOTO K, SATO M, SATO T, TOMITA Y, MAEDA R, et al. Clinical and epidemiologic factors related to subsequent wheezing after virus-induced lower respiratory tract infections in hospitalized pediatric patients younger than 3 years. Eur J Ped 2014; 173: 959-66.

5.- SIGURS N, BJARNASON R, SIGURBERGSSON F, KJELLMAN B. Respiratory syncytial virus bronchiolitis in infancy is an important risk factor for asthma and allergy at age 7. Am J Respir Crit Care Med 2000; 161: 1501-7.

6.- KUSEL M M, DE KLERK N H, KEBADZE T, VOHMA V, HOLT P G, JOHNSTON S L, et al. Early-life respiratory viral infections, atopic sensitization, and risk of subsequent development of persistent asthma. J Allergy Clin Immunol 2007; 119: 1105-10.

7.- BOUSQUET J, HEINZERLING L, BACHERT C, PAPADOPOULOS N G, BOUSQUET P J, BURNEY P $\mathrm{G}$, et al. Practical guide to skin prick tests in allergy to aeroallergens. Allergy 2012; 67: 18-24.

8.- JACKSON D J, EVANS M D, GANGNON R E, TISLER C J, PAPPAS T E, LEE W M, et al. Evidence for a causal relationship between allergic sensitization and rhinovirus wheezing in early life. Am J Respir Crit Care Med 2012; 185: 281-5.

9.- SAGLANI S. Viral infections and the development of asthma in children. Therapeutic Adv Infect Dis 2013; 1: 139-50.

10.- CASTRO-RODRÍGUEZ J A, RAMÍREZ A M, TOCHE P, PAVON D, PÉREZ M A, GIRARDI G, et al. Clinical, functional, and epidemiological differences between atopic and nonatopic asthmatic children from a tertiary care hospital in a developing country.Ann Allergy Asthma Immunol 2007; 98: 239-44.

11.- ARBES S J JR, GERGEN P J, VAUGHN B, ZELDIN D C. Asthma cases attributable to atopy: results from the Third National Health and Nutrition Examination Survey.J Allergy Clin Immunol 2007; 120: 1139-45.

12.- SLY P D, BONER A L, BJÖRKSTEN B, BUSH A, CUSTOVIC A, EIGENMANN P A, et al. Early identification of atopy in the prediction of persistent asthma in children. Lancet 2008; 372: 1100-6.

13.- OKAYAMA Y. Cellular and humoral immunity of virusinduced asthma. Front Microbiol 2013; 4: 1-7.

14.- COX D W, LE SOUËF P N. Rhinovirus and developing lung. Paediatr Respir Rev 2014; 15: 268-74.

15.- DÍAZ P V, GAGGERO A A, PINTO R A, MAMANI R, UASAPUD P A, BONO M R. Aumento de interleuquinas proinflamatorias y de cortisol plasmático en bronquiolitis por virus respiratorio sincicial: relación con la gravedad de la infección. Rev Med Chile 2013; 141: 574-81.

16.- PINTO R A, ARREDONDO S M, BONO M R, GA- 
GGERO A A, DÍAZ P V. T Helper 1/T Helper 2 cytokine imbalance in respiratory syncytial virus infection is associated with increased endogenous plasma cortisol. Pediatrics 2006; 117: 878-86.

17.- DÍAZ P V, PINTO R A, MAMANI R, UASAPUD $P$ A, BONO M R, GAGGERO A A, et al. Increased expression of the glucocorticoid receptor $\beta$ in infants with RSV bronchiolitis. Pediatrics 2012; 130: 804-11.

18.- CASTRO-RODRÍGUEZ J A, HOLBERG C J,
WRIGHT A L, MARTÍNEZ F D. A clinical index to define risk of asthma in young children with recurrent wheezing. Am J Respir Crit Care Med 2000; 162: 1403 6.

19.- VORAPHANI N, STERN D A, WRIGHT A L, GUERRA S, MORGAN W J, MARTÍNEZ F D. Risk of Current Asthma among Adult Smokers with Respiratory Syncytial Virus Illnesses in Early Life. Am J Respir Crit Care Med 2014; 190: 392-8.

Correspondencia a:

Dr. Guillermo Zepeda F.

Profesor Asistente

Depto. de Pediatría y Cirugía Infantil,

Facultad de Medicina, Campus Norte,

Universidad de Chile.

Hospital Roberto del Río. Santiago de Chile.

Email: gezepeda@med.uchile.cl 